\title{
Probing TeV Physics through Neutron-Decay Matrix Elements
}

\author{
Huey-Wen Lin* and Saul D. Cohen \\ Department of Physics, University of Washington, Seattle, WA 98195-1560
}

Tanmoy Bhattacharya, Rajan Gupta and Anosh Joseph

Theoretical Division, Los Alamos National Laboratory, Los Alamos, NM 87545, USA

\begin{abstract}
We study the presence of new scalar and tensor interactions beyond the Standard Model at the $\mathrm{TeV}$ scale via high-precision calculations of low-energy observables with lattice gauge theory. We compute matrix elements of isovector bilinear operators between nucleon states to high precision using valence clover fermions on dynamical $N_{f}=2+1+1$ HISQ configurations generated by the MILC Collaboration. These ensembles are available at multiple lattice spacings and sea-quark masses yielding pion masses as light as $140 \mathrm{MeV}$, allowing us to remove the large systematic uncertainties associated with the chiral and continuum extrapolations. In this presentation, we will show results from 2 lattice spacings, 0.12 and $0.09 \mathrm{fm}$ with pion masses less than $310 \mathrm{MeV}$.
\end{abstract}

The XXIX International Symposium on Lattice Field Theory

July 10-16, 2011

Lake Tahoe, California

*speaker

†NT@UW-11-26 
Precision measurements of nucleons provide constraints on the Standard Model (SM) and can discern the signatures predicted for particles beyond the Standard Model (BSM). Knowing the Standard Model inputs to nucleon matrix elements will be necessary to constrain the couplings of dark matter candidates such as the neutralino, to relate the neutron electric dipole moment to the $\mathrm{CP}$-violating theta parameter, or to search for new TeV-scale particles though non- $V-A$ interactions in neutron beta decay. At high energy, the LHC has been built to discover the mechanism for electroweak symmetry breaking and to probe certain potential new BSM particles which appear at the $\mathrm{TeV}$ scale, such as some candidates for dark matter. Such high-energy colliders in general are well suited to production of high-mass particles directly at their resonances and for probing processes with cross-sections that scale with energy. However, there are many low-energy precision experiments that also have the ability to probe BSM physics. These generally fall into two classes: experiments that can very precisely measure physics that is precisely predicted by the SM, such as muon $g-2$, the proton weak charge, and flavor-changing processes involving the CKM matrix. Another class of experiments can look for tiny signals in places that the SM says should be either undetectably small or exactly zero, such as the neutron electric dipole moment, or non$V-A$ contributions to neutron beta decay. These low-energy experiments, which usually involve detections through interactions with nuclear matter, will require understanding of the low-energy nonperturbative properties of QCD. Using lattice gauge theory, we can nonperturbatively calculate the QCD path integral on a supercomputer; with increasing computational resources and improved algorithm, we are now entering a new era for precision calculation. In this proceeding, I will discuss a few representative areas in which lattice QCD (LQCD) can contribute to the search for BSM physics, emphasizing suppressed operators in neutron decay, and outline prospects for future development.

There are many opportunities for LQCD to provide important inputs to probe BSM physics with nucleons. For example, the strange contributions to the proton's scalar and spin densities are important inputs for spin-independent and -dependent cross-sections. Recent evidence suggests that the composition of dark matter must be cold, with velocities that are non-relativistic, giving it a Compton wavelength above the width of a proton. One promising candidate for dark matter is the supersymmetric particle called the neutralino. If dark matter is neutralinos, they will interact with nuclei at low energy through the Higgs particle. Many groups have developed techniques to investigate $\sigma_{s}=m_{s}\langle N|\bar{s} s| N\rangle$; a recent global fit to current dynamical lattice data (weighted by lattice spacing, lightest $m_{\pi}$, dynamical strange and other quality factors, shown on the left-hand side of Fig. 1 in Ref. [1]) gives $\sigma_{s}^{\mathrm{LQCD}}=47(8) \mathrm{MeV}$. The value can be input into theoretical calculations of cross-section constraints from various models; an example of the application to the CMSSM can be found in Ref. [2].

The neutron electric dipole moment also plays an important role in constraining many BSM theories. Although experiments do not have the necessary precision to measure the SM value $\left(10^{-30} e \cdot \mathrm{cm}\right)$, many BSM models predict values that are higher than the experimental upper bounds, so these models have been ruled out. This includes some parts of the parameter space for certain SUSY models. LQCD calculations can study such a dipole moment by adding a CPodd $\theta$-term to the Lagrangian and studying the electromagnetic form factor $F_{3}$, by looking at the energy difference for two different spin states of the nucleon at zero momentum under an external static, uniform electric field, or by examining the product of the anomalous magnetic moment of 
neutron $F_{2}\left(q^{2}=0\right)$ and $\tan (2 \alpha)$ (by QCDSF) in a CP-violating system. The collected dynamical lattice-QCD results on $d_{n}$ are shown on the right-hand side of Fig. 1 in Ref. [1], and the combined extrapolation to the physical pion mass gives $d_{n}^{\mathrm{LQCD}}=-0.015(5) \theta e \cdot \mathrm{fm}$. More updated and precise calculations from various groups are currently in progress.

A third example of probing BSM with nucleons (and the main focus for the remainder of this proceeding) is to look for signatures of contributions due to new scalar or tensor interactions in neutron beta decay [3]. The notion is quite similar to how Fermi theory led to the discovery of the electroweak interaction and its bosons. Beta decay was originally explained by Fermi by adding a new term to the fundamental Lagrangian describing 4-fermion interactions. Such a theory introduces a coupling, the Fermi constant, that has units of inverse-energy squared with an energy scale around $100 \mathrm{GeV}$. As it turns out, the Fermi theory is a low-energy effective theory approximating the electroweak theory, which has 3 vector bosons, the $W$ 's and $Z$. The theory was later probed in high-energy proton-antiproton experiments at CERN, and the new particles were found with resonances around the scale predicted by this interpretation of the Fermi theory. We can imagine that new particles beyond the Standard Model can be predicted in just such a way: by first detecting the low-energy effective operators and later directly probing them in a high-energy experiment.

The neutron beta-decay Hamiltonian contains the original $V-A$ current for the leptons and the quarks, and in addition it will contain terms corresponding to the new BSM physics:

$$
H_{\mathrm{eff}}=G_{F}\left(J_{V-A}^{\mathrm{lept}} \times J_{V-A}^{\mathrm{quark}}+\sum_{i} \varepsilon_{i}^{\mathrm{BSM}} \hat{O}_{i}^{\mathrm{lept}} \times \hat{O}_{i}^{\text {quark }}\right),
$$

where $G_{F}$ is the Fermi constant, $J_{V-A}$ indicates the left-handed current of the indicated particle, and the sum includes operators with novel chiral structure. So in the context of our theory, new operators will enter with the coefficients $\varepsilon$ that are related to the $\mathrm{TeV}$ scale of the particles. The leptonic part is understandable using analytic techniques, but the quark operator in the context of the nucleon will introduce some unknown coupling constants, $g_{T}=\left\langle n\left|\bar{u} \sigma_{\mu v} d\right| p\right\rangle, g_{S}=\langle n|\bar{u} d| p\rangle$, which are nonperturbative functions of the nucleon structure, described in the SM by QCD. Any deviation from the SM $V-A$ current coming from the new scalar and tensor interactions in the effective theory will require knowledge of the couplings $g_{S}$ and $g_{T}$ to understand. For more details about experimental and theoretical work on this subject, we refer readers to the proceedings of this conference, Refs. [4, 5] and an upcoming paper [3], and references within.

To make a precision calculation of these low-energy coupling constants, we need ensembles with multiple lattice spacings whose lightest pion masses are below $200 \mathrm{MeV}$, have $M_{\pi} L \leq 4$, and we must collect high statistics with currently available computational resources. The MILC ensembles with $N_{f}=2+1+1$ highly improved staggered quarks (HISQ) [6] contain lattice spacings ranging from 0.15 to $0.045 \mathrm{fm}$ and with multiple physical-mass ensembles $m_{u, d} / m_{s}=1 / 27[7,8,9,10]$, which would help to remove the uncertainties due to chiral extrapolation. We chose to use HISQ sea ensembles and clover fermion action in the valence sector to work on these nucleon quantities. We HYP-smeared the gauge fields and the quark sources are Gaussian-smeared. "Coherent" sources are used to calculate the three-point functions, after checking for consistency with the traditional approach. The source-sink separation is around $1.2 \mathrm{fm}$; further investigations on the effects of the excited-state containmination systematics regarding to the source-sink separation are on the way. 

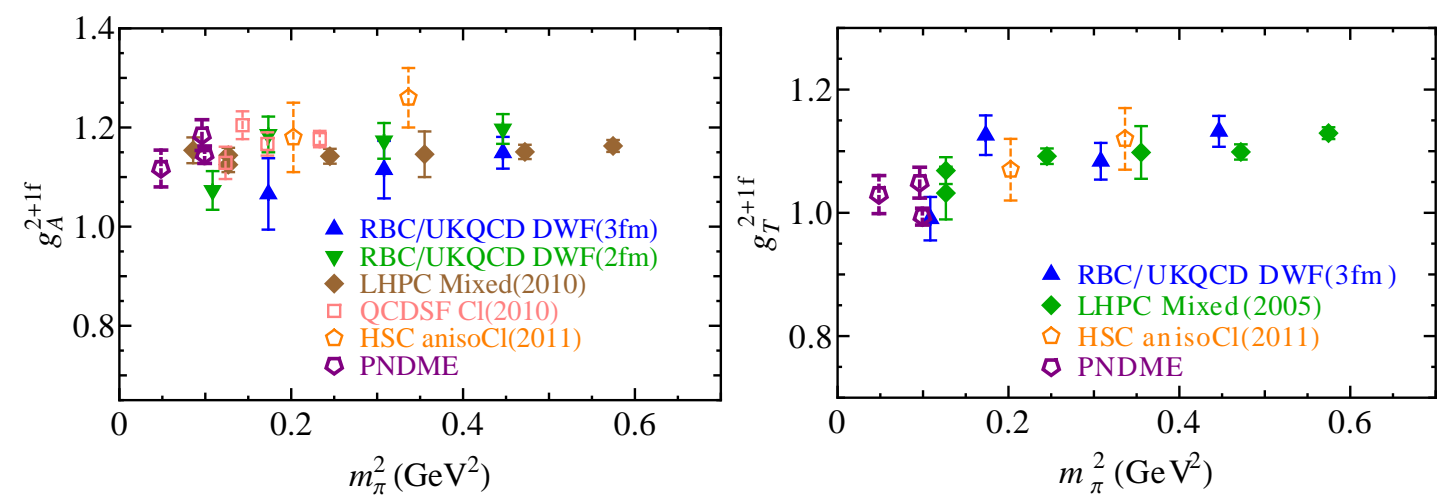

Figure 1: Summary of the lattice $N_{f}=2+1$ results on $g_{A}$ and $g_{T}$ as functions of $m_{\pi}^{2}$. Our preliminary results using HISQ lattices are shown in the purple downward pentagons.

The charges are tree-level tadpole renormalized for the moment, and a nonperturbative renormalization (NPR) procedure is on the way. In this proceeding, we present results from 3 ensembles: $0.12 \mathrm{fm}$ with $310-$ and $220-\mathrm{MeV}$ pions and $0.09 \mathrm{fm}$ with $310-\mathrm{MeV}$ pions.

Figure 1 shows a world summary of $g_{A}$ and $g_{T}$ from previous $N_{f}=2+1$ lattice calculations, along with ours (downward purple pentagons). Our charges from the $0.12 \mathrm{fm}$ ensembles are consistent with the results from RBC/UKQCD (who have used NPR) and LHPC (who use "scaled" nonperturbative $Z_{A}$ and perturbative renormalization for other operators) whose ensembles has around the same lattice spacing. For the rest of the proceeding, we will focus on the $g_{S, T}$ and their application to neutron beta decay.

The tensor charge $\left(g_{T}\right)$ is also known as the zeroth moment of transversity; one can study it experimentally through processes such as SIDIS (semi-inclusive deep inelastic scattering), probing the nucleon structure. Here is an example of results obtained from HERMES and COMPASS. Experimentally, one can extract the contribution from individual quarks as a function of the quark momentum fraction $x$, at certain $Q^{2}$; in this case $Q^{2}=2.4 \mathrm{GeV}^{2}$. To obtain $g_{T}$, one needs to integrate over all possible $x$, that is, from 0 to 1, through an interpolation of the few available $x$ at each $Q^{2}$. After combining a few experiments, the best experimental estimate (using model inputs) for this quantity is obtained at $Q^{2}=0.8 \mathrm{GeV}^{2}$ (instead of $Q^{2}=0$ as in the correct definition of $g_{T}$ ): $0.77_{-0.24}^{+0.18}$ with combined uncertainty around $25 \%[11,12,13,14]$. Furthermore, the experimental value is not a gold-plated quantity like the nucleon axial charge. It requires data over a wide range of $x$, and the low- $x$ (and high- $x$ ) values are not well known, although this could be improved in the future experiments. There are also theoretical models that attempt to make estimates of the tensor charge, such as Nambu-Jona-Lasinio model [15] and the chiral quark soliton model [16]; unfortunately, they are not consistent with each other. And using QCD sum rules, still yields a large uncertainty [17]. The other important ingredient for the new interactions is the scalar charge $g_{S}$. There are no experimental measurements of this quantity, and the theoretical estimations (from different model approximations) give rather loose bounds to the quantity: $0.25 \leq g_{S} \leq 1$.

Lattice calculation of $g_{T}$ and $g_{S}$ is rather straightforward. The tensor charge has been studied a few times in the past using $N_{f}=2+1$ dynamical ensembles in lattice QCD, as summarized in the left-hand side of Fig. 2. We take the 2+1-flavor results by the RBC [18] and LHPC [19], and re- 

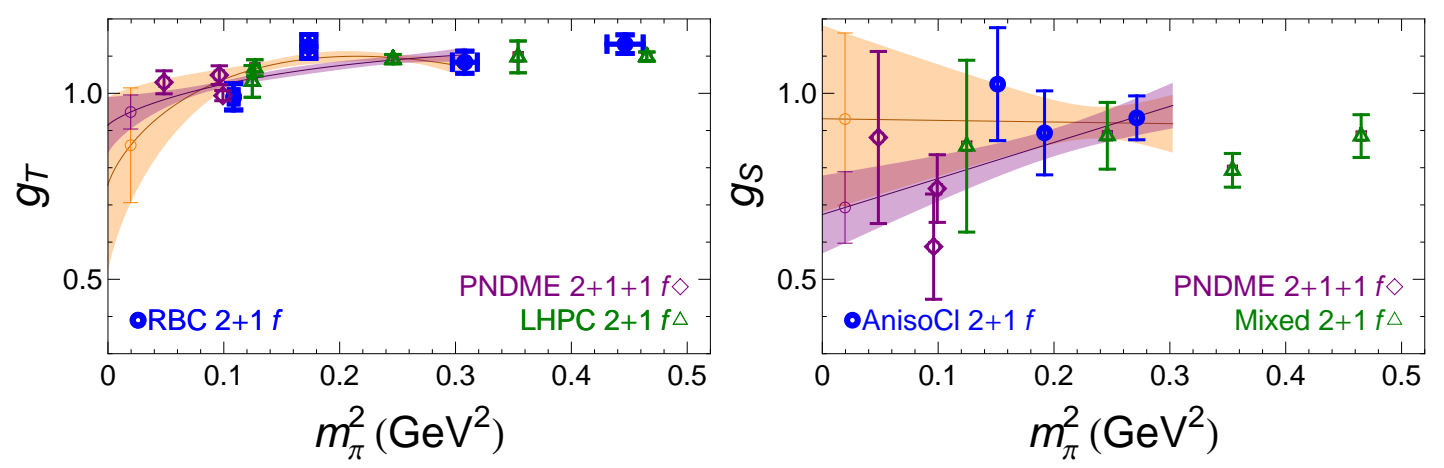

Figure 2: Global analysis of all $N_{f}=2+1$ lattice calculations of $g_{T}$ (left) and $g_{S}$ (right). The leftmost points are the extrapolated values at the physical pion mass. The two bands show different extrapolations in the pion mass: with and without the lighter PNDME collaboration points from this work; the final uncertainty due to the chiral extrapolation is significantly improved.

cently by PNDME (this work), and make a global plot as a function of pion mass squared. $g_{S}$, on the other hand, has not been much studied; we collect new calculations, shown on the right-hand side of Fig. 2 using various $N_{f}=2+1$ lattice actions. All of these results, by different collaborations, using different fermions actions, (but at the same lattice spacing) are generally in good agreement, although the errorbars shown here contain only statistical error. We further globally analyze all the available lattice data (with a pion mass cut at $550 \mathrm{MeV}$ ), including (excluding) the lightest available from PNDME, around $220 \mathrm{MeV}$, shown as the purple (orange) band in Fig. 2, using the chiral formulation given in Ref. [20] and linear ansatz for tensor and scalar charges respectively extrapolation to the physical pion mass. We see that the PNDME points greatly constrain the uncertainty due to chiral extrapolation in both cases and obtain $g_{T}^{\mathrm{LQCD}}=0.95(5)$ and $g_{S}^{\mathrm{LQCD}}=0.69(9)$.

Finally, we can combine the low-energy tensor and scalar charges with experimental data to determine the allowed region of the parameter space for scalar and tensor BSM couplings (denoted $\varepsilon)$. We can combine the current knowledge of $g_{S, T}$ and the existing experimental data for the nuclear beta decay $0^{+} \rightarrow 0^{+}$transition and others, such as $\beta$ symmetry in Gamow-Teller ${ }^{60} \mathrm{Co}$, longitudinal polarization ratio between Fermi and Gamow-Teller transitions in ${ }^{114} \mathrm{In}$, positron polarization in polarized ${ }^{107}$ In and beta-neutrino correlation parameters in nuclear transitions. Using the $g_{S, T}$ from the model estimations and combining with the existing nuclear experimental data, we get the constraints shown the outermost band on the right-hand side of Fig. 3. There is an ongoing ultra-cold neutron (UCN) experiment studying neutron beta decay at LANL to look for deviations from the SM in the Fierz term and the neutrino asymmetry parameter to the level of $10^{-3}$ by 2013. Combining those expected data and existing measurements, and again, using the model inputs of $g_{S, T}$, we see the uncertainties in $\varepsilon_{S, T}$ are significantly improved. This shows the importance of the precision experimental inputs. Finally, using our present lattice-QCD values of the scalar and tensor charges, combined with the expected 2013 precision of experimental bounds on deviation of these neutron-decay parameters from their SM values, we found the constraints on $\varepsilon_{S, T}$ are further improved, shown as the innermost region. These upper bounds on the effective couplings $\varepsilon_{S, T}$ correspond to lower bounds for the scales $\Lambda_{S, T}$ at 2.9 and $5.6 \mathrm{TeV}$, respectively, for new physics in these channels. 

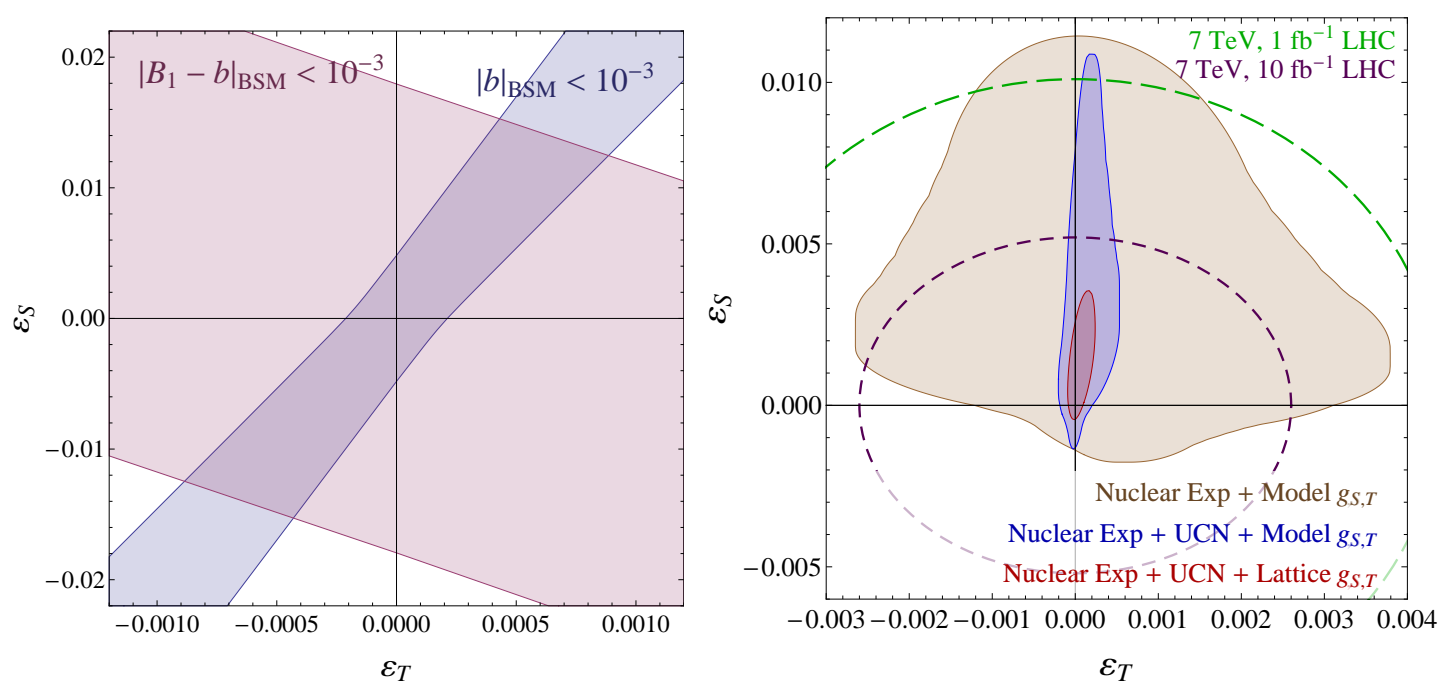

Figure 3: (left) $\varepsilon_{\mathrm{S}, \mathrm{T}}$ using currently available lattice calculations on $g_{\mathrm{S}, \mathrm{T}}$ with anticipated $b_{\mathrm{BSM}}$ and $B_{\mathrm{BSM}}-$ $b_{\mathrm{BSM}}$ within $10^{-3}$ expected from UCNB/b at LANSCE by 2013. (right) $\varepsilon_{S^{-}} \varepsilon_{T}$ allowed parameter region using different experimental and theoretical inputs. The green and purple dashed lines are the constraints from LHC current bounds and near-term expectation.

How do the constraints from high-energy experiments compare? As demonstrated in Ref. [3], neither CDF nor D0 are sufficient to provide useful constraints. We can estimate the $\varepsilon_{S, T}$ constraints from LHC current bounds and near-term expectations through effective Lagrangian

$$
\mathscr{L}=-\frac{\eta_{S}}{\Lambda_{S}^{2}} V_{\mathrm{ud}}(\bar{u} d)\left(\bar{e} P_{L} v_{e}\right)-\frac{\eta_{T}}{\Lambda_{T}^{2}} V_{\mathrm{ud}}\left(\bar{u} \sigma_{u, d} d\right)\left(\bar{e} \sigma_{u, d} P_{L} v_{e}\right)
$$

where $\eta_{S, T}= \pm 1$. By looking at events with high transverse mass from CMS/ATLAS in the $e v+X$ channel and comparing with the SM $W$ background, we estimated 90\%-C.L. constraints on $\eta_{S, T}$ based on the current LHC run, $\sqrt{s}=7 \mathrm{TeV} \mathscr{L}=1 \mathrm{fb}^{-1}$ (the green line) and for a near-future run $\sqrt{s}=7 \mathrm{TeV} \mathscr{L}=10 \mathrm{fb}^{-1}$ (the purple dashed line) on the right-hand side of Fig. 3. More details can be found in Ref. [3].

If experiments can measure these BSM signals to be nonzero, combining with our precision $g_{S, T}$ calculations, we will be able to set a range of possible masses for potential new particles that may be probed directly by high-energy colliders, such as the LHC. If experiment cannot rule out the SM, then we will provide lower bounds for the scale of new physics in these channels, which will help to rule out certain classes of BSM models. As experimental precision on these quantities improve, it is important that the precision of these neglected lattice matrix elements do not become an obstacle to probing the precision frontier for new physics. We will accomplish this in the future by improving our calculation with physical pion masses, nonperturbative renormalization, improved statistics and continuum extrapolation.

\section{Acknowledgments}

We thank MILC for sharing their HISQ lattices with us. These calculations were performed using the Chroma software suite [21]. The initial tuning was done on Hyak clusters at the University 
of Washington eScience Institute, using hardware awarded under NSF grant PHY-09227700. The production runs were done using USQCD resources. The speaker is supported by the DOE grant DE-FG02-97ER4014.

\section{References}

[1] H.-W. Lin (2011), 1109.2542.

[2] J. Giedt, A. W. Thomas, and R. D. Young, Phys. Rev. Lett. 103, 201802 (2009), 0907.4177.

[3] T. Bhattacharya et al., forthcoming .

[4] R. Gupta et al. (PNDME), this conference procceding .

[5] T. Bhattacharya et al. (PNDME), this conference procceding .

[6] E. Follana et al. (HPQCD), Phys. Rev. D75, 054502 (2007), hep-lat/0610092.

[7] A. Bazavov et al., PoS LATTICE2010, 320 (2010), 1012.1265.

[8] A. Bazavov et al. (MILC), Phys. Rev. D82, 074501 (2010), 1004.0342.

[9] A. Bazavov et al. (MILC), PoS LAT2009, 123 (2009), 0911.0869.

[10] A. Bazavov et al. (MILC), PoS LATTICE2008, 033 (2008), 0903.0874.

[11] M. Anselmino et al., Nucl. Phys. Proc. Suppl. 191, 98 (2009), 0812.4366.

[12] M. Alekseev et al. (COMPASS Collaboration), Phys. Lett. B673, 127 (2009), 0802.2160.

[13] M. Diefenthaler (HERMES Collaboration) pp. 579-582 (2007), 0706.2242.

[14] R. Seidl et al. (Belle Collaboration), Phys. Rev. D78, 032011 (2008), 0805.2975.

[15] I. Cloet, W. Bentz, and A. W. Thomas, Phys. Lett. B659, 214 (2008), 0708.3246.

[16] M. Wakamatsu, Phys. Lett. B653, 398 (2007), 0705.2917.

[17] H.-X. He and X.-D. Ji, Phys. Rev. D52, 2960 (1995), hep-ph/9412235.

[18] Y. Aoki et al., Phys. Rev. D82, 014501 (2010), 1003.3387.

[19] R. Edwards, G. Fleming, P. Hagler, J. W. Negele, K. Orginos, et al., PoS LAT2006, 121 (2006), hep-lat/0610007.

[20] W. Detmold, W. Melnitchouk, and A. W. Thomas, Phys. Rev. D66, 054501 (2002), hep-lat/0206001.

[21] R. G. Edwards and B. Joo (SciDAC), Nucl. Phys. Proc. Suppl. 140, 832 (2005), hep-lat/0409003. 